\title{
An update on the epidemiology, imaging and therapy of brain metastases
}

\author{
Maciej Mielczarek , Anna Kubica', Mateusz Szylberg ${ }^{1}$, Karolina Zielińska', Jakub Przybył, \\ Agata Sierzputowska², Marek Harat ${ }^{1}$
}

${ }^{1} 10^{\text {th }}$ Military Research Hospital and Polyclinic, Bydgoszcz, Poland

2Ludwik Rydygier Collegium Medicum in Bydgoszcz, Nicolaus Copernicus University in Toruń, Poland

Introduction. The incidence of brain metastases (BM) is rapidly increasing, with most cases occurring in patients aged 50-80 years and in 10-40\% of patients with systemic neoplastic disease. The Graded Prognostic Assessment (GPA) is the most impartial prognostic method, according to which the average survival rate of patients with brain metastases is only 7.18 months

Purpose. To present a systematic review of the currently available evidence-based literature on the epidemiology, diagnosis, and treatment of BM.

Methods. The authors searched PubMed up to March 2020 using the phrases "brain metastases", "brain metastasis surgery", and "brain metastases treatment", which returned 65 citations.

Conclusions. The choice of imaging and therapy for brain metastases remains a significant clinical problem. MRI, including T1, T1 + C, T2, FLAIR, and SWI sequences, is the most sensitive method for solitary BM detection, while other techniques such as spectroscopy, perfusion imaging, or fractional anisotropy contribute to diagnosis precision and neurological deficit avoidance in cases eligible for surgery. According to current treatment algorithms, three main methods are used to manage BM: surgery, chemotherapy, and radiotherapy, depending on the expected effect and the patient's clinical condition. Surgery is most often used, offering neurological deficit remission in 60 to $90 \%$ of patients. Most chemotherapeutics do not cross the blood-brain barrier, so immunotherapy with antibodies such as pembrolizumab and ipilimumab, as well as antineoplastic vaccines, are a promising therapeutic prospect.

NOWOTWORY J Oncol 2020; 70, 3: 111-117

Key words: brain, cancer, metastasis, imaging, therapy, surgery, immunotherapy

\section{Key messages}

- In spite of recent improvements in diagnostics and treatment, there are few data describing the incidence and prognosis of patients with BM.

- The diagnosis and treatment of BM still pose a major challenge for all oncology-related specializations.

- Traditional surgical excision has been used since the beginning of BM management and remains effective.
- Currently available targeted therapies still require further study but there are promising advances in the field.

\section{Introduction}

Cancer is the second most common cause of death in developed countries, with intracranial brain metastases (BM) the most common neurological complication of systemic cancer. BM are associated with significant morbidity and mortality [1, 2],

\section{How to cite:}

Mielczarek M, Kubica A, Szylberg M, Zielińska K, Przybył J, Sierzputowska A, Harat M. An update on the epidemiology, imaging and therapy of brain metastases. NOWOTWORY J Oncol 2020; 70: 111-117. 
and the number of patients admitted with BM has increased markedly over past few decades [3]. Amongst patients with systemic neoplastic disease, about half will develop BM during the whole period of the management of the disease [4].

Brain metastases appear as round, ring-enhancing lesions, usually at the white and grey matter junction, on magnetic resonance imaging (MRI) or computed tomography (CT). MRI is more sensitive at detecting BM than CT, detecting 20\% more multiple metastases [1]. Patients presenting with headaches, seizures, or other neurological deficits are imaged by MRI with gadolinium contrast. However, only 30\% of MRIs conducted at the time of neurologic diagnosis reveal solitary lesions [5]. Magnetic resonance spectroscopy (MRS) can contribute new information about BM metabolism and the surrounding edema.

Brain metastases are refractory to existing systemic therapies, mainly due to a unique set of brain growth factors promoting resistance and inefficient drug delivery due to the blood-brain barrier [6]. Treatment algorithms are based on prognosis and whether the aim of management is symptom palliation, prolongation of survival, or both [6]. As with all cancer management, there are three core therapeutic approaches to BM: surgery, radiotherapy, and chemotherapy. However, only a few chemotherapies penetrate the blood-brain barrier, a longstanding situation that has hampered progress in BM therapy [5]. However, newer innovative therapeutic strategies are being developed based on targeted molecular and immunological approaches. The newest clinical studies have shown that the central nervous system (CNS) should be treated as a different (but not isolated) immunological environment variant that requires the application of targeted therapy, such as immune check-point inhibitors (ICI) [4].

In spite of recent improvements in diagnostics and treatment, there are few data describing the incidence and prognosis of patients with BM. The aim of this review was to summarize the incidence, diagnosis, treatment possibilities, and prognoses of patients with BM.

\section{Methods}

The authors searched PubMed up to March 2020 using the phrases "brain metastases", "brain metastasis surgery", and "brain metastases treatment", which returned 65 citations.

\section{Epidemiology}

The incidence of brain metastases is increasing and, due to diagnostic problems, their exact incidence is unknown. The data on brain metastases incidence that are available in the literature are disparate and difficult to compare, originating from epidemiological, clinical, and autopsy studies.

An early population-based analysis of brain metastases conducted between 1954 and 1963 in Iceland reported an annual incidence of BM of 2.8 affected individuals per 100,000; the incidence proportion of metastatic brain tumors in all patients with primary systemic malignancies was less than
20\% [7]. Ten years later, a US (United States) study reported an annual incidence of BM of 8.3 per 100,000 [8], while a population-based review of the Metropolitan Detroit Cancer Surveillance System from 1973 to 2001 found an incidence proportion of brain metastasis among all patients with systemic malignancies to be $9.6 \%$. This probably represents an underestimate, because the authors only included lung, melanoma, breast, renal, and colorectal cancers [7]. A Swedish healthcare registry study from 1987 to 2006 found that the annual incidence rate of hospitalization for BM doubled during this period from 7 to 14 persons per 100,000. While these population-based studies were performed in different populations and without standardized treatment, they appear to indicate an increase in incidence [9]. This trend towards an increase in brain metastases incidence has also been observed in neurosurgical departments. In a retrospective cohort study of the Nationwide Inpatient Sample, there was an increase in the annual number of surgical resections for BM from 3900 in 1988 to 7000 in 2000 [10].

However, every population-based study must be regarded as an underestimate because of neurologically silent metastases. Autopsy studies have shown that up to a quarter of patients diagnosed with cancer have BM before death $[11,12]$. It is estimated that there will be 1,762,450 new cancer cases in the US in 2019, 440,612 of whom will develop BM during their lifetime. Schouten and colleagues ${ }^{8}$ reported that approximately $70 \%$ of cancer patients develop BM within the first year after diagnosis, meaning that at least 308,428 BM cases are expected in 2019 in the US. Compared to 258,886 reported BM cases in the same database in 2009: this represents an increase of about 20\% [13, 14].

The origin and histotype of the primary cancer and improved survival rates from diagnosis are major factors affecting BM incidence. Sophisticated imaging techniques and better management of diffused neoplastic diseases (that leads to longer overall survival) are the main dimensions that contribute to the increase of BM incidence [15]. In order of decreasing frequency, the majority of BM arise from cancer of the lung, breast, kidney, and gastrointestinal (Gl) tract and from melanomas [16].

The estimated annual incidence of lung cancer in 2019 was 228,150 [14], and lung cancer is the most frequent (30$60 \%$ ) source of BM. Between $17 \%$ and $65 \%$ of patients with primary lung cancer develop BM $[9,16]$, most commonly from small cell lung cancer and adenocarcinomas. Lung cancer frequently presents with brain metastases as the first symptom of systemic disease. The median interval between initial cancer diagnosis and identification of a BM is shortest for lung cancer and ranges from 2 to 9 months, with $91 \%$ of patients with lung cancer being diagnosed with brain metastases within one year of initial diagnosis $[9,16]$. The presence of an epidermal growth factor receptor (EGFR) mutation is associated with a higher incidence of brain metastases in patients with non-small cell lung cancer (NSCLC) [17]. From 
these data, 138,792 new lung cancer BMs are expected to be diagnosed in the US in 2019.

In contrast to lung cancer, brain metastases arising from breast cancer arise later in the disease course, a median of 2-3 years after the initial diagnosis. The estimated annual new incidence of breast cancer in 2019 is 271,270 [14]. Breast cancer is the most common source of BM in women, accounting for 5-30\% of all metastatic brain tumors in women and occurring in up to $30 \%$ of women with primary breast cancer [18]. Younger age, human epidermal growth factor receptor 2 (HER2) positivity, estrogen receptor (ER) negativity, high tumor grade, high proliferative activity, and high burden of metastatic disease are all risk factors for BM in breast cancer [19-21]. Graesslin et al. developed a nomogram to predict subsequent BM in patients with breast cancer without brain metastatic disease based on these risk factors [22]. From these data, $\sim 56,966$ new breast cancer brain metastases will be diagnosed in the US in 2019.

The incidence of melanoma is also increasing, with an estimated 96,480 new cases in 2019 and a higher incidence in male patients. Approximately $37 \%$ of patients with stage IV melanoma develop BM, with autopsy series reporting an incidence as high as 90\%. Melanoma BM are associated with the poorest outcome of all cancers. Although NCCN (The National Comprehensive Cancer Network) guidelines recommend a brain MRI for stage III (regional) and IV (metastatic) melanoma, Mustafa et al. suggested cranial evaluation for earlier-stage patients, even for localized disease [23].

There are estimated to be 101,420 new colorectal cancer cases in the US in 2019, again more commonly in men than in women [14]. Approximately $10 \%$ of patients with stage IV colorectal cancer have brain metastases, and the median interval between initial colorectal cancer diagnosis and identification of a BM is approximately 2-3 years [16]. We estimate the caseload of new brain metastases using the data in table $\mathrm{I}$.

\section{Imaging diagnosis and morphology}

T1, T1 + C, T2, FLAIR, and SWI sequences are commonly used to study patients with possible BM. Gadolinium-based contrast agents (GBCA) are available for use in clinical applications of MRI. Increasing the GBCA dose increases the sensitivity for small $(<5 \mathrm{~mm}$ ) lesions [24] and, similarly, increasing MRI field strength improves metastasis detection; contrast dose appears to have greater impact than field strength, although half-dose contrast at double resonance field strength is reportedly superior to full dose at normal resonance field strength [25]. Due to the development of nephrogenic systemic fibrosis, high doses of gadolinium are now avoided, and in light of US Food and Drug Administration (FDA) warnings against the use of high-dose gadolinium, other potential enhancement methods for BM are needed. Increasing the time delay (by 15 minutes) between contrast injection and acquisition has been shown to result in at least one additional lesion being detected in 43\% of patients with BM [26]. Moreover, the volume of metastatic lesions detected is greater after time delay [27]. A delay of 20 minutes appears to be optimal for maximizing the detection of small lesions. The distribution of contrast agent in BM can vary; typical well-defined ring-enhancing lesions are only apparent in $15 \%$ of BM. With this in mind, Dolgushin et al. subclassified BM into five groups: (I) target, with decay in the center of the tumor (26.9\%); (II) heterogeneous, with multiple decay fields (13.9\%); (III) ring (15.3\%); (IV) ring and tissue (13.1\%); and (V) homogeneous (30.8\%) [28].

Although brain metastases typically exhibit well-defined margins delimited by a glial pseudo-capsule, recent studies have demonstrated that there is often surrounding brain infiltration. Brain colonization by metastatic cells could be promoted by the glial defense system in the adjacent brain parenchyma, neutralization of which seems to be a prerequisite for brain parenchymal colonization in vivo [29]. The impact of astrocytes on chemotherapy resistance and tumor cell proliferation has been reported [30, 31]. In an ex vivo organotypic brain slice co-culture mode, astrocytes and microglia accumulated at the metastasis/brain parenchyma interface, formed multiple protrusions, and interacted with the immortalized benign non-CNS (central nervous system) epithelial cells to subsequently induce apoptosis in these cells [32,33]. Siam et al.

Table I. Estimated new cancer cases and deaths by sex, United States, 2019 [13]

\begin{tabular}{|c|c|c|c|c|c|c|c|}
\hline & \multicolumn{3}{|c|}{ Estimated new cases } & \multicolumn{3}{|c|}{ Estimated deaths } & \multirow{2}{*}{$\begin{array}{c}\text { Estimated number of BM } \\
\text { General }\end{array}$} \\
\hline & General & Male & Female & General & Male & Female & \\
\hline All sites & $1,762,450$ & 870,970 & 891,480 & 606,880 & 321,670 & 285,210 & 308,428 \\
\hline Colon & 101,420 & 51,690 & 49,730 & 51,020 & 27,640 & 23,380 & 10,142 \\
\hline Lung & 228,150 & 116,440 & 111,710 & 142,670 & 76,650 & 66,020 & 138,792 \\
\hline Melanoma & 96,480 & 57,220 & 39,260 & 7,230 & 4,740 & 2,490 & 60,782 \\
\hline Breast & 271,270 & 2,670 & 268,600 & 42,260 & 500 & 41,760 & 56,966 \\
\hline Kidney & 73,820 & 44,120 & 29,700 & 14,770 & 9,820 & 4,950 & Not enough data to estimate \\
\hline Primary Brain Tumors & 23,820 & 13,410 & 10,410 & 17,760 & 9,910 & 7,850 & \\
\hline
\end{tabular}


proposed four types of metastatic infiltration: type 0 , displacing growth without infiltration - non-infiltrating cancer cells with a significant glial reaction (typical for renal cell cancer); type 1, cluster/cohort infiltration - strands invade the adjacent brain parenchyma with detached infiltrating cohorts and clusters sometimes found in the Virchow-Robin space, but also without contact with the blood vessels; type 2, diffuse infiltration - single cells or mini-spheres infiltrating the brain parenchyma; and type 3, angio-cooptive - typically, infiltration into the adjacent brain parenchyma takes place along preexisting blood vessels (typical for melanoma) [33].

These studies strongly suggest that microscopic total resection should be favored over gross total resection to achieve clinical benefits. The tissue of the cavity wall should, where possible, be resected beyond the glial pseudo-capsule. Fluorescence-guided resections with preoperative 5 -aminolevulinic acid (5-ALA) seem to be clinically beneficial. Recent studies have shown that material obtained from the fluorescent tissue of the cavity walls contains tumor cells in 33\% of cases [34]. Patients with an infiltrative phenotype have poor prognostic outcomes. In addition, Ki-67 has a generally poor prognostic value, only being prognostic in NSCLC and RCC (renal cell carcinoma) [35].

The gold standard test for detection of BMs is MRI, since infiltrative areas can be missed with standard morphologic imaging. Perfusion imaging, spectroscopy, and diffusion-weighted imaging enhance the diagnostic accuracy, and diffusion-weighted imaging, apparent diffusion coefficient imaging, and fractional anisotropy imaging are used for tractography, which can prevent deficits after surgery.

\section{Treatment of brain metastases}

Brain metastases are a very common complication in oncology patients and pose a therapeutic challenge. The presence of BM usually heralds a dismal prognosis. The most objective evaluation of prognosis is the Graded Prognostic Assessment (GPA), which calculates an average survival rate for patients with BM of only 7.18 months [36]. The aforementioned index poses a useful tool while estimating a diagnostic-specific prognosis for patients with BM [37]. Specific factors taken into consideration vary, depending on the particular diagnosis and are strictly specified for the five neoplasms that most often cause BM: breast, renal, lung and gastrointestinal cancers, as well as melanoma. The GPA for different diagnoses can consist of several aspects: Karnofsky performance score, age, presence of extracranial metastases, number of BM and tumor subtype [38] (tab. II-IV). Diagnosis-specific Graded Prognostic Assessment (DS-GPA) is an extended version of the GPA index. DS-GPA includes primary tumor type, gene status and subtypes of breast cancer (tab. II-IV).

However, the molecular and histological features of the tumors have a big impact on survival rates. For instance, patients with breast cancer brain metastases and positive HER2 status
Table II. Significant factors taken into consideration while estimating prognosis for particular neoplasms most frequently causing BM $[7,39,40]$

\begin{tabular}{|ll}
\hline Type of neoplasm & Significant prognostic factors \\
\hline Lung carcinoma & - Age \\
& - KPS \\
& - Number of BM \\
& - EGFR mutation \\
\hline Breast cancer (adenocarcinoma) & - Age remodelling \\
& - KPS \\
& - ER, PR, HER2 mutations \\
\hline Hypernephroma & - KPS \\
\hline Melanoma & - Number of BM \\
& - KPS \\
& - Number of BM \\
\hline Colon adenocarcinoma & - BRAF mutation \\
& - KPS \\
& - Age \\
\hline & - Number of BM \\
& \\
\hline
\end{tabular}

Table III. Graded prognostic assessment

\begin{tabular}{lcc}
\hline Factors & $>60$ & Score \\
\hline Age & $50-59$ & 0 \\
& $<50$ & 0.5 \\
\hline KPS & $<70$ & 1.0 \\
\hline Number of CNS metastases & $70-80$ & 0 \\
\hline Extracranial metastases & $90-100$ & 0.5 \\
\hline & $2-3$ & 1.0 \\
\hline & 1 & 0 \\
\hline & present & 0.5 \\
\hline
\end{tabular}

Table IV. Median survival time for the GPA

\begin{tabular}{|lc|}
\hline GPA score & MST [months] \\
\hline $0-1$ & 2.6 \\
\hline $1.5-2.5$ & 3.8 \\
\hline 3.0 & 6.9 \\
\hline $3.5-4.0$ & 11.0 \\
\hline
\end{tabular}

can survive over five years with multimodal therapy and good control of the systemic disease [41]. In melanoma or NSCLC, the median survival is poor, and even young patients with good life quality generally survive less than a year [35]. Management should consider tumor histology where possible, available 
elective treatment, the patient's age, Karnofsky performance status, the volume of brain metastases, and extracranial disease activity [41]. The main treatment methods include surgery, whole brain radiotherapy (WBRT), stereotactic radiosurgery, and chemotherapy.

Whole brain radiotherapy alone has been compared to surgery plus WBRT for the treatment of newly-diagnosed brain metastases [42, 43], with good quality evidence of the benefits from the application of the combined regimen (median survival 4-6 and 10 months, respectively). Furthermore, disease-free survival and CNS recurrence rates are improved with multimodal treatment [41].

Most surgery produces good clinical results. $60-90 \%$ of operable patients benefit from symptom remission [44]. The application of perioperative techniques such as preoperative MRI, neuronavigation, and intraoperative electrophysiological techniques have extended the range of possible surgeries, improved the ability to perform radical resection, and improved safety [45]. Only 20\% of non-radical tumor removal cases are visible on postoperative MRI. A lack of gross total resection of metastases is the biggest risk factor for local recurrence [46]. From the surgeon's perspective, the choice of technique and the impact of the operative method used on neurological function are important considerations, although a large retrospective study examining surgical effectiveness and safety showed that it is possible to remove a single brain metastasis with no neurological decline, even in eloquent regions [47].

Recent reports have shown that surgery of two or even three brain metastases has similar effectiveness to the surgical treatment of a single metastasis, albeit in patients with good overall neurological condition and well-controlled systemic disease [48].

Over the past few years, stereoradiosurgery (SRS) has emerged as a promising method [49]. Stereoradiosurgery allows the very precise irradiation of a tumor mass, with the convergence of superinduced rays accumulating the dose in one place and sparing the surrounding brain tissue. Stereoradiosurgery is particularly useful for the treatment of small metastases and lesions that are hard to reach by surgery [50]. The aforementioned method can be divided into stereotactic fractionated radiotherapy (SFRT) and single-dose SRS. Due to recommended dose limits, some tumors must be treated with fractionated doses. Single-dose SRS applied to no more than three brain lesions has been shown to achieve local control, defined as a lack of growth or a decrease in tumor mass after one year, in about $80-90 \%$ of cases [51]. The effects of treatment on metastases considered to be radio-resistant (melanomas or hypernephromas) are similar to those that are radio-sensitive. Another advantage of SRS is the possibility of using it on the elderly (>80 years old) [51-53]. Reports on WBRT combined with SRS have only shown improved outcomes in patients with high GPA (3.5-4) (WBRT combined with SRS vs. WBRT alone was 21.0 vs. 10.3 months). In patients with a worse GPA, there is no major difference between WBRT and WBRT combined with SRS [54]. Of note, SRS toxicity does not depend on the total number of metastases but only on their aggregate volume [53]. This method can also be used in combination with preoperative immunotherapy with anlotinib (anti-VEGF - vascular endothelial growth factor). In a multicenter clinical study, Wang et al. have proven that anti-VEGF factors substantively reduced brain edema, which led to better surgery tolerance and the enhancement of SRS effects [49].

Nowadays, more clinicians acknowledge the necessity of postoperative adjuvant methods. There are two complementary therapies which are becoming the newest standards: WBRT and SRS delivered to the resection cavity [56]. Moravan et al., in their study, claim that both methods have similar overall survival (OS), while SRS is marked by two significant advantages: longer cognitive - deterioration-free survival (dysfunction after 6 months in patients who underwent SRS vs. WBRT: $52 \%$ vs. $85 \%$ ) and major reduction of local tumor recurrence (12-month period exempt from recurrence: $72 \%$ vs. $43 \%$ comparing postoperative SRS and no adjuvant therapies applied, respectively) [56]. The newest NCCN guidelines advocate the application of SRS alone as an adjuvant therapy amongst patients whose total metastases volume is limited; addition of WBRT significantly aggravates neurological and cognitive declines [23, 57]. For cavities larger than $5 \mathrm{~cm}$ total in volume, single-fraction adjuvant SRS should not be applied [23].

In view of the presence of the blood-brain barrier, metastatic tumors do not respond well to systemic chemotherapy, which is used to control systemic disease. However, there are some exceptions. EGFR-positive NSCLC radiologically responds to erlotinib, gefitinib, and osimertinib [45], targeted EGFR inhibitors. In addition, several other pathways are being examined as therapeutic targets, such as PI3K/Akt/mTOR (phosphoinositide 3-kinase/ protein kinase B/ mammalian target of rapamycin pathway), HER3 (human epidermal growth factor receptor 3), VEGF and polymerase inhibitors (PARPi).

Classical chemotherapeutics avoid the blood-brain barrier in three different ways, all of which are associated with barrier transport mechanisms. (1) Absorbing transcytosis, which is a phenomenon that describes the connection between positively charged molecules with brain endothelial cells (on which there are negatively charged caveolae or corrugations covered with clathrin). Chemotherapeutics, being positively charged, increase the distribution through the blood-brain barrier. Unfortunately, the occurrence of negatively polarized cell membranes throughout the body contributes to the significant toxicity of this method [55]. (2) Paclitaxel transport with the use of transport proteins turned out to be effective in animal models [58]. (3) Receptor transport, where endocytic transport is contingent on the ligand. This method allows the transport of large molecules. Attempts to exploit this method include the use of monoclonal antibodies and the approach has been applied to Alzheimer's and Parkinson's disease [59]. 
In view of the immunological privilege of the brain, immunotherapy represents a challenging but promising therapeutic. Most recommended targeted therapies are those which use anti-VEGF factors and checkpoint inhibitors, such as pembrolizumab [60]. Wang et al. suggest that inhibitors of PD-1 and PD-L1 (programmed death receptor-1/programmed death ligand 1) activate the antineoplastic effect of the T cells located in the tumor microenvironment (TME), previously inhibited by the tumor. Therefore, in comparison to methods routinely used in managing $\mathrm{BM}$, those therapies feature reduced neurotoxicity [38]. What's more, there are strong reasons to believe that immunocytes are able to comprehensively relocate into or out of the CNS [38]. For example, pembrolizumab (an anti-PD-1 immunotherapy) had a positive impact on intracranial melanoma or NSCLC metastases in 20-30\% of cases [38]. Furthermore, in $55 \%$ of melanoma treatments, therapy with nivolumab and ipilimumab (anti-PD1 and CTLA-4- cytotoxic T-lymphocyte-associated protein 4) led to intracranial tumor remission [60, $62,38,63]$. As a result, the latest NCCN guidelines recommend the application of nivolumab as a routine therapy for patients with initial or recurrent stage III/V melanoma disease [23]. In a 2018 phase III clinical trial KEYNOTE-189, patients with NSCLC and BM were divided into two groups: one treated with chemotherapy alone and the other, whose treatment consisted of chemotherapy and pembrolizumab. The second group elicited a markedly better survival outcome than the patients on monotherapy [61].

There is also recent research in antineoplastic vaccines, since overexpression of the antigens that participate in carcinogenesis causes immunization. The most promising vaccine for patients with brain metastases in stage I/II of clinical research is PERCELLVAC3. Moreover, there is interest in the use of oncolytic viruses, which results in an immunological response not only to the virus but also the tumor, and which has been tested with anti-PD-L1 therapy and resulted in improved outcomes [64].

\section{Conclusions}

The incidence of metastatic brain tumors is increasing. The diagnosis and treatment of BM still pose a major challenge for all oncology-related specializations. Traditional surgical excision has been used since the beginning of BM management and remains effective. Currently available targeted therapies still require further study but there are promising advances in the field.

Conflict of interest: none declared

\author{
Maciej Mielczarek \\ 10th Military Research Hospital and Polyclinic \\ ul. Powstańców Warszawy 5 \\ 85-001 Bydgoszcz, Poland \\ e-mail:maciejmielczarek87@gmail.com
}

Received: 1 Jan 2020

Accepted: 22 Apr 2020

\section{References}

1. Cagney D, Martin A, Catalano P, et al. Incidence and prognosis of patients with brain metastases at diagnosis of systemic malignancy: a population-based study. Neuro-Oncology. 2017; 19(11): 1511-1521, doi: $10.1093 /$ neuonc/nox077.

2. Nayak L, Lee E, Wen P. Epidemiology of Brain Metastases. Curr Oncol Rep. 2011; 14(1): 48-54, doi: 10.1007/s11912-011-0203-y.

3. Smedby KE, Brandt L, Bäcklund ML, et al. Brain metastases admissions in Sweden between 1987 and 2006. Br J Cancer Suppl. 2009; 101(11): 1919-1924, doi: 10.1038/sj.bjc.6605373.

4. Giacomo ADi, Valente M, Cerase A, et al. Immunotherapy of brain metastases: breaking a "dogma". J Exp Clin Cancer Res. 2019; 38(1), doi: 10.1186/s13046-019-1426-2.

5. Muldoon L, Soussain C, Jahnke K, et al. Chemotherapy Delivery Issues in Central Nervous System Malignancy: A Reality Check. J Clin Oncol. 2007; 25(16): 2295-2305, doi: 10.1200/jco.2006.09.9861.

6. Lim M, Puttick S, Houston Z, et al. Innovative Therapeutic Strategies for Effective Treatment of Brain Metastases. Int J Mol Sci. 2019; 20(6): 1280, doi: 10.3390/ijms20061280.

7. Barnholtz-Sloan J, Sloan A, Davis F, et al. Incidence Proportions of Brain Metastases in Patients Diagnosed (1973 to 2001) in the Metropolitan Detroit Cancer Surveillance System. J Clin Oncol. 2004; 22(14): 2865-2872, doi: 10.1200/jco.2004.12.149.

8. Walker $A E$, Robins $M$, Weinfeld FD. Epidemiology of brain tumors: The national survey of intracranial neoplasms. Neurology. 1985; 35(2): 219-219, doi: 10.1212/wnl.35.2.219.

9. Schouten L, Rutten J, Huveneers $\mathrm{H}$, et al. Incidence of brain metastases in a cohort of patients with carcinoma of the breast, colon, kidney, and lung and melanoma. Cancer. 2002; 94(10): 2698-2705, doi: 10.1002/ cncr.10541.

10. Barker F. Craniotomy for the resection of metastatic brain tumors in the U.S., 1988-2000. Cancer. 2004; 100(5): 999-1007, doi: 10.1002/ cncr.20058.

11. Aronson S, Garcia J, Aronson B. Metastatic neoplasms of the brain Their frequency in relation to age. Cancer. 1964; 17(5): 558-563, doi: 10.1002/1097-0142(196405)17:5<558::aid-cncr2820170503>3.0.co;2-e.

12. Posner J. Intracranial metastases from systemic cancer. Adv Neurol. 1978; 19: 579-592.

13. Fox $B$, Cheung V, Patel $A$, et al. Epidemiology of Metastatic Brain Tumors Neurosurgery Clinics. 2011; 22(1): 1-6, doi: 10.1016/j.nec.2010.08.007.

14. Siegel R, Miller K, Jemal A. Cancer statistics, 2018. CA Cancer J Clin. 2018; 68(1): 7-30, doi: 10.3322/caac.21442.

15. Wanleenuwat $P$, Iwanowski $P$. Metastases to the central nervous system: Molecular basis and clinical considerations. J Neurol Sci. 2020; 412: 116755, doi: 10.1016/j.jns.2020.116755.

16. Suki D. The Epidemiology of Brain Metastasis. Intracranial Metastases. : 20-34, doi: 10.1002/9780470753064.ch2.

17. Porta R, Sanchez-Torres JM, Paz-Ares L, et al. Brain metastases from lung cancer responding to erlotinib: the importance of EGFR mutation. Eur Respir J. 2010; 37(3): 624-631, doi: 10.1183/09031936.00195609.

18. Cheng $X$, Hung MC. Breast cancer brain metastases. Cancer Metastasis Rev. 2007; 26(3-4): 635-643, doi: 10.1007/s10555-007-9083-x.

19. Hicks D, Short S, Prescott N, et al. Breast Cancers With Brain Metastases are More Likely to be Estrogen Receptor Negative, Express the Basal Cytokeratin CK5/6, and Overexpress HER2 or EGFR. Am J Surg Pathol. 2006; 30(9): 1097-1104, doi: 10.1097/01.pas.0000213306.05811.b9.

20. Pestalozzi $\mathrm{BC}$, Zahrieh D, Price $\mathrm{KN}$, et al. Identifying breast cancer patients at risk for Central Nervous System (CNS) metastases in trials of the International Breast Cancer Study Group (IBCSG). Ann Oncol. 2006; 17(6): 935-944, doi: 10.1093/annonc/mdl064.

21. Tham $\mathrm{YL}$, Sexton $\mathrm{K}$, Kramer R, et al. Primary breast cancer phenotypes associated with propensity for central nervous system metastases. Cancer. 2006; 107(4): 696-704, doi: 10.1002/cncr.22041.

22. Graesslin O, Abdulkarim B, Coutant C, et al. Nomogram to Predict Subsequent Brain Metastasis in Patients With Metastatic Breast Cancer. J Clin Oncol. 2010; 28(12): 2032-2037, doi: 10.1200/jco.2009.24.6314.

23. Network NCC. NCCN Clinical Practice Guidelines in Oncology-Melanoma Version 1.; 2020: 2019.

24. Togao O, Hiwatashi A, Yamashita K, et al. Additional MR contrast dosage for radiologists' diagnostic performance in detecting brain metastases: a systematic observer study at 3 T. Jpn J Radiol. 2014; 32(9): 537-544, doi: 10.1007/s11604-014-0342-9.

25. Krautmacher C, Willinek W, Tschampa H, et al. Brain Tumors: Full- and Half-Dose Contrast-enhanced MR Imaging at 3.0 T Compared with 1.5 T-Initial Experience. Radiology. 2005; 237(3): 1014-1019, doi: 10.1148/radiol.2373041672 
26. Kushnirsky M, Nguyen V, Katz J, et al. Time-delayed contrast-enhanced MRI improves detection of brain metastases and apparent treatment volumes. J Neurosurg. 2016; 124(2): 489-495, doi: 10.3171/2015.2.jns141993.

27. Cohen-Inbar Or, Xu Z, Dodson B, et al.Time-delayed contrast-enhanced MRI improves detection of brain metastases: a prospective validation of diagnostic yield. J Neurooncol. 2016; 130(3): 485-494, doi: 10.1007/ s11060-016-2242-6.

28. Dolgushin M, Kornienko V, Pronin I. Brain Metastases: Advanced Neuroimaging. Springer International Publishing 2017

29. Valiente M, Obenauf A, Jin X, et al. Serpins Promote Cancer Cell Survival and Vascular Co-Option in Brain Metastasis. Cell. 2014; 156(5): 1002-1016, doi: 10.1016/j.cell.2014.01.040.

30. Fidler I. The role of the organ microenvironment in brain metastasis. Semin Cancer Biol. 2011; 21(2): 107-112, doi: 10.1016/j.semcancer.2010.12.009.

31. Lin Q, Balasubramanian K, Fan D, et al. Reactive Astrocytes Protect Melanoma Cells from Chemotherapy by Sequestering Intracellular Calcium through Gap Junction Communication Channels. Neoplasia. 2010; 12(9): 748-754, doi: 10.1593/neo.10602.

32. Berghoff A, Rajky O, Winkler F, et al. Invasion patterns in brain metastases of solid cancers. Neuro-Oncology. 2013; 15(12): 1664-1672, doi: 10.1093/neuonc/not112.

33. Siam L, Bleckmann A, Chaung HN, et al. The metastatic infiltration at the metastasis/brain parenchyma-interface is very heterogeneous and has a significant impact on survival in a prospective study. Oncotarget. 2015; 6(30): 29254-29267, doi: 10.18632/oncotarget.4201.

34. Kamp MA, Grosser P, Felsberg J, et al. 5-aminolevulinic acid (5-ALA)-induced fluorescence in intracerebral metastases: a retrospective study. Acta Neurochir (Wien). 2012; 154(2): 223-228.

35. Berghoff A, Ilhan-Mutlu A, Dinhof $C$, et al. Differential role of angiogenesis and tumour cell proliferation in brain metastases according to primary tumour type: analysis of 639 cases. Neuropathol Appl Neurobiol. 2015; 41(2): e41-e55, doi: 10.1111/nan.12185.

36. Sperduto P, Kased N, Roberge D, et al. Summary Report on the Graded Prognostic Assessment: An Accurate and Facile Diagnosis-Specific Tool to Estimate Survival for Patients With Brain Metastases. J Clin Oncol. 2012; 30(4): 419-425, doi: 10.1200/jco.2011.38.0527.

37. Kim $\mathrm{H}$, Lee $\mathrm{K}, \mathrm{Heo} \mathrm{M}$, et al. The prognostic factors influencing overall survival in uterine cervical cancer with brain metastasis. Korean J Intern Med. 2019; 34(6): 1324-1332, doi: 10.3904/kjim.2018.051.

38. Wang S, Hu C, Xie F, et al. Use of Programmed Death Receptor-1 and/ or Programmed Death Ligand 1 Inhibitors for the Treatment of Brain Metastasis of Lung Cancer. Onco Targets Ther. 2020; 13: 667-683, doi: 10.2147/ott.s235714.

39. Schouten L, Rutten J, Huveneers $\mathrm{H}$, et al. Incidence of brain metastases in a cohort of patients with carcinoma of the breast, colon, kidney, and lung and melanoma. Cancer. 2002; 94(10): 2698-2705, doi: 10.1002/ cncr.10541.

40. Sperduto $P$, Fang $P, L i ~ J$, et al. Estimating survival in patients with gastrointestinal cancers and brain metastases: An update of the graded prognostic assessment for gastrointestinal cancers (GI-GPA). Clin Transl Radiat Oncol. 2019; 18: 39-45, doi: 10.1016/j.ctro.2019.06.007.

41. Soffietti R, Chiavazza C, Rudà R. Imaging and clinical end points in brain metastases trials. CNS Oncology. 2017; 6(4): 243-246, doi: 10.2217/ cns-2017-0017.

42. Mintz A, Kestle J, Rathbone M, et al. A randomized trial to assess the efficacy of surgery in addition to radiotherapy in patients with a single cerebral metastasis. Cancer. 1996; 78(7): 1470-1476, doi: 10.1002/ (sici) 1097-0142(19961001)78:7<1470::aid-cncr14>3.0.co;2-x.

43. Patchell R, Tibbs P, Walsh J, et al. A Randomized Trial of Surgery in the Treatment of Single Metastases to the Brain. N Engl J Med. 1990; 322(8): 494-500, doi: 10.1056/nejm199002223220802.

44. Pessina F, Navarria P, Cozzi L, et al. Role of Surgical Resection in Patients with Single Large Brain Metastases: Feasibility, Morbidity, and Local Control Evaluation. World Neurosurg. 2016; 94: 6-12, doi: 10.1016/j. wneu.2016.06.098.

45. Kelly W, Shah N, Subramaniam D. Management of Brain Metastases in Epidermal Growth Factor Receptor Mutant Non-Small-Cell Lung Cancer. Front Oncol. 2018; 8, doi: 10.3389/fonc.2018.00208.
46. Kamp M, Rapp M, Slotty P, et al. Incidence of local in-brain progression after supramarginal resection of cerebral metastases. Acta Neurochir (Wien). 2015; 157(6): 905-911, doi: 10.1007/s00701-015-2405-9.

47. Patel A, Suki D, Hatiboglu M, et al. Impact of surgical methodology on the complication rate and functional outcome of patients with a single brain metastasis. J Neurosurg. 2015; 122(5): 1132-1143, doi: 10.3171/2014.9.jns13939.

48. Nahed B, Alvarez-Breckenridge C, Brastianos P, et al. Congress of Neurological Surgeons Systematic Review and Evidence-Based Guidelines on the Role of Surgery in the Management of Adults With Metastatic Brain Tumors. Neurosurgery. 2019; 84(3): E152-E155, doi: 10.1093/ neuros/nyy542.

49. Wang $Y$, Wang $X$, Guan $Y$, et al. Stereotactic radiosurgery combined with anlotinib for limited brain metastases with perilesional edema in non-small cell lung cancer: Rvision-001 study protocol. Thorac Cancer. 2020; 11(5): 1361-1364, doi: 10.1111/1759-7714.13386.

50. Baumert B, Rutten I, Dehing-Oberije C, et al. A pathology-based substrate for target definition in radiosurgery of brain metastases. Int J Radiat Oncol Biol Phys. 2006; 66(1): 187-194, doi: 10.1016/j.ijrobp.2006.03.050.

51. Lippitz B, Lindquist C, Paddick I, et al. Stereotactic radiosurgery in the treatment of brain metastases: The current evidence. Cancer Treat Rev. 2014; 40(1): 48-59, doi: 10.1016/j.ctrv.2013.05.002.

52. Karlsson B, Hanssens $P$, Wolff $R$, et al. Thirty years' experience with Gamma Knife surgery for metastases to the brain. J Neurosurg. 2009; 111(3): 449-457, doi: 10.3171/2008.10.jns08214.

53. Watanabe $S$, Yamamoto $M$, Sato $Y$, et al. Stereotactic radiosurgery for brain metastases: a case-matched study comparing treatment results for patients 80 years of age or older versus patients 65-79 years of age. J Neurosurg. 2014; 121(5): 1148-1157, doi: 10.3171/2014.6.jns132790.

54. Sperduto P, Shanley R, Luo X, et al. Secondary Analysis of RTOG 9508, a Phase 3 Randomized Trial of Whole-Brain Radiation Therapy Versus WBRT Plus Stereotactic Radiosurgery in Patients With 1-3 Brain Metastases; Poststratified by the Graded Prognostic Assessment (GPA). Int J Radiat Oncol Biol Phys. 2014; 90(3): 526-531, doi: 10.1016/j. ijrobp.2014.07.002.

55. Hervé F, Ghinea N, Scherrmann JM. CNS Delivery Via Adsorptive Transcytosis. The AAPS Journal. 2008; 10(3): 455-472, doi: 10.1208/ s12248-008-9055-2.

56. Moravan M, Fecci $P$, Anders $C$, et al. Current multidisciplinary management of brain metastases. Cancer. 2020; 126(7): 1390-1406, doi: 10.1002/cncr.32714.

57. Network NCC. NCCN Clinical Practice Guidelines in Oncology- NonSmall Cell Lung Cancer Version 3. ; 2020.

58. Jiang YZ, Jiménez J, Ou K, et al. Hemodynamic Disturbed Flow Induces Differential DNA Methylation of Endothelial Kruppel-Like Factor 4 Promoter In Vitro and In Vivo. Circ Res. 2014; 115(1): 32-43, doi: 10.1161/ circresaha.115.303883.

59. Yu YJ, Zhang Y, Kenrick M, et al. Boosting Brain Uptake of a Therapeutic Antibody by Reducing Its Affinity for a Transcytosis Target. Sci Transl Med. 2011; 3(84): 84, doi: 10.1126/scitransImed.3002230.

60. Fallah J, Ahluwalia M. The role of immunotherapy in the management of patients with renal cell carcinoma and brain metastases. Ann Transl Med. 2019; 7(S8): S313-S313, doi: 10.21037/atm.2019.10.31.

61. Kamath S, Kumthekar P. Immune Checkpoint Inhibitors for the Treatment of Central Nervous System (CNS) Metastatic Disease. Front Oncol. 2018; 8, doi: 10.3389/fonc.2018.00414.

62. Tawbi H, Forsyth P, Algazi A, et al. Combined Nivolumab and Ipilimumab in Melanoma Metastatic to the Brain. N Engl J Med. 2018; 379(8): 722-730, doi: 10.1056/nejmoa1805453.

63. Lehrer $\mathrm{E}$, McGee H, Sheehan J, et al. Integration of immuno-oncology with stereotactic radiosurgery in the management of brain metastases. J Neurooncol. 2020, doi: 10.1007/s11060-020-03427-6.

64. Du W, Seah I, Bougazzoul O, et al. Stem cell-released oncolytic herpes simplex virus has therapeutic efficacy in brain metastatic melanomas. Proceedings of the National Academy of Sciences. 2017; 114(30): E6157-E6165, doi: 10.1073/pnas.1700363114. 